\title{
The Role of Nicotinic Acetylcholine Receptors in the Primary Reinforcing and Reinforcement-Enhancing Effects of Nicotine
}

\author{
Matthew I Palmatier*, I,2, Xiu Liu', Anthony R Caggiula', Eric C Donny' and Alan F Sved ${ }^{2}$ \\ 'Department of Psychology, University of Pittsburgh, Pittsburgh, PA, USA; ${ }^{2}$ Department of Neuroscience, University of Pittsburgh, Pittsburgh, \\ PA, USA
}

\begin{abstract}
The primary reinforcing effects of nicotine are mediated by the drugs action at central nervous system nicotinic acetylcholine receptors (nAChRs). Although previous studies have demonstrated that nicotine potently enhances responding for non-pharmacological stimuli, the role of nAChRs in this reinforcement-enhancing effect is not known. The two reinforcement-related effects of nicotine can be dissociated in a paradigm that provides concurrent access to drug infusions and a non-pharmacological visual stimulus (VS). The present study characterized the role of nAChRs in the primary reinforcing effect of nicotine and the reinforcement-enhancing effect of nicotine. For rats with access to VS (VS-Only), nicotine (NIC-Only), both reinforcers contingent upon one response (NIC+ VS) or both reinforcers contingent upon separate responses (2-Lever), unit dose-response relationships (0,30,60, or $90 \mu \mathrm{gg} / \mathrm{kg} / \mathrm{infusion}$, free base) were determined over a 22-day acquisition period. Expression of the two reinforcement-related effects of nicotine was manipulated by pharmacological antagonism of nAChRs (I mg/kg mecamylamine, subcutaneous, 5-min before the session) or by substituting saline for nicotine infusions (ie extinction) over a series of seven test sessions. Unit dose manipulations yielded an inverse dose-response relationship for active lever responding in the NIC + VS group. The dose-response relationships for rats with independent access to each reinforcer (2-Lever group) were relatively flat. For the 2-Lever group, acute mecamylamine challenge blocked the reinforcementenhancing effects of nicotine, VS-lever responding decreased to basal levels on the first day of mecamylamine treatment or saline substitution (to the level of the VS-Only group). In contrast, nicotine-lever responding decreased gradually over the 7-day testing period (similar to saline extinction). The two reinforcement-related effects of nicotine are mediated by nAChRs but can be dissociated by acute and chronic profiles.

Neuropsychopharmacology (2007) 32, I098- I 108. doi: I 0. I 038/sj.npp. I 30 I228; published online 8 November 2006
\end{abstract}

Keywords: smoking; self-administration; extinction; reinforcement; dose-response; nicotinic acetylcholine receptor

\section{INTRODUCTION}

Although tobacco smoke contains more than 4000 compounds, nicotine has been identified as the major tobacco ingredient that maintains chronic smoking behavior (USDHHS, 1988). This conclusion is supported by the numerous failed attempts of tobacco companies to market products with decreased nicotine yield (Jaffe, 1990) and pre-clinical findings in which operant responses (ie leverpresses) are strengthened or sustained by nicotine delivery in humans, non-human primates, canines, and rodents (eg Corrigall and Coen, 1994; Goldberg et al, 1981; Harvey et al, 2004; Risner and Goldberg, 1983).

Despite the undeniable role of nicotine in human smoking, non-nicotine stimuli have become an increasingly

*Correspondence: Dr MI Palmatier, Department of Psychology, University of Pittsburgh, 3137 Sennott Square, 210 S Bouquet Street, Pittsburgh, PA 15260, USA, Tel: + | 412 624 7345, Fax: + I 412624 8558, E-mail: mip 16@pitt.edu

Received 6 April 2006; revised 28 August 2006; accepted 29 August 2006

Online publication: I5 September 2006 at http://www.acnp.org/ citations/Npp09 I506060226/default.pdf investigated contributor to tobacco addiction (Rose, 2006). In human smoking, nicotine delivery occurs in conjunction with a number of non-pharmacological stimuli that are inextricable from smoking behavior (eg throat irritation, smell, and taste of smoke, see Rose and Levin, 1991). Indeed, the recent work of Rose and co-workers has demonstrated that sensorimotor stimuli associated with smoking are particularly important to smoking behavior (Rose et al, 2003) and subjective ratings of liking (Rose et al, 2000). For example, puffing denicotinized cigarettes has a stronger satiating effect on ad lib smoking than pulsed infusions of nicotine (Rose et al, 2003). Similarly, exclusion of non-nicotine stimuli in a rodent model of nicotine selfadministration can decrease responding for the drug to levels that are only moderately higher than controls that self-administer vehicle (Caggiula et al, 2002). Thus, nonnicotine stimuli can be considered a critical component of rodent self-administration models that are designed to reveal the relationship between two essential elements of smoking in humans.

Recent advances in the rodent nicotine self-administration paradigm have led us to propose a new model of nicotine reinforcement (Chaudhri et al, 2006a), in which the 
drug has two dissociable actions on operant responding. First, nicotine can function as a primary reinforcer of operant behavior; rats will respond for nicotine in the absence of non-pharmacological stimuli, albeit at very low rates (eg Caggiula et al, 2002). Second, nicotine enhances responding for non-pharmacological stimuli that have some reinforcing value, an effect that was initially demonstrated by removing the contingency between drug delivery and operant responding (Donny et al, 2003). Thus, nicotine not only supports operant behavior (ie serves as a primary reinforcer) but also inflates the incentive value of nonnicotine reinforcers. The dissociation of these two effects was recently confirmed in a study that separated the operant response required to earn nicotine infusions from the response required to earn presentation of a nonpharmacological reinforcer, which in this case was a compound visual stimulus (VS; Palmatier et al, 2006). Response rates from groups of rats that could obtain the two reinforcers concurrently by pressing different levers (ie NIC or VS) were compared to response rates from groups that pressed one lever for both reinforcers (NIC + VS) or for each reinforcer alone (NIC-Only or VS-Only). The concurrent access group (henceforth referred to as the 2-Lever group) responded for nicotine at rates expected by primary reinforcement, that is, comparable to rats self-administering nicotine-alone (NIC-Only group). In addition, concurrent access to nicotine had an enhancing effect on VS-derived reinforcement; responding for the VS was enhanced relative to rats that could only earn the non-pharmacological stimulus (VS-Only group), and comparable to rats receiving access to both stimuli for making a single operant response (NIC + VS group).

This dissociation between the two reinforcement-related effects of nicotine provides a unique opportunity to investigate their behavioral and pharmacological substrates. For example, manipulations that decrease nicotine seeking are not contaminated by changes to the unconditional effects of nicotine on the stimulus (ie reinforcementenhancing effect) or by changes in conditional value that have accrued via pairings of nicotine and VS (ie conditioned reinforcement). Therefore, the present study was designed to characterize the role of nicotinic acetylcholine receptors (nAChRs) in both the primary reinforcing and reinforcement-enhancing effects of nicotine. To do so, we varied the concentration of nicotine delivered for each infusion earned (unit dose, 30-90 $\mu \mathrm{g} / \mathrm{kg} / \mathrm{infusion}$, free base) to determine if changing the intensity and/or duration of drug action at nAChRs enhanced or attenuated responding for nicotine, VS, or both. Next, nAChRs were antagonized by systemic administration of a non-selective, non-competitive antagonist (mecamylamine) before each of seven consecutive daily sessions (MEC-EXT) in which rats had continued access to nicotine. Response rates from the mecamylamine tests were compared to a saline extinction condition in which rats received vehicle pretreatment and vehicle infusions instead of nicotine (SAL-EXT).

\section{METHODS}

Subject: Male Sprague-Dawley rats (Harlan Farms, IN) weighing 174-200 g on arrival were housed individually in hanging wire mesh cages. Rats were housed in a temperature- and humidity-controlled colony room on a reverse $12: 12 \mathrm{~h}$ light: dark cycle. Unrestricted access to food and water was allowed for 3 days after their arrival. Food access was subsequently restricted to $20 \mathrm{~g}$ /day allowing limited growth (approximately $20 \mathrm{~g} /$ week) throughout the remainder of the study (Donny et al, 1995).

Apparatus: All experimental sessions were conducted in $25 \times 31 \times 8(\mathrm{w} \times 1 \times \mathrm{h}) \mathrm{cm}$ operant conditioning chambers (BRS/LVE Model RTC-020, MD; see Donny et al, 1995 for further details). The VS consisted of 1-s illumination of a white cue light located directly above the 'VS' or 'active' lever, followed by 1-min deactivation of the house light. During each session, rats were connected to a swivel system that delivered intravenous infusions while allowing nearly unrestricted movement in the chamber.

Drugs: Nicotine hydrogen tartrate salt and mecamylamine hydrochloride (Sigma, St Louis, MO) were dissolved in $0.9 \%$ saline. The nicotine solution $\mathrm{pH}$ was adjusted to $7.0( \pm 0.2)$ with a dilute $\mathrm{NaOH}$ solution. Depending on dose condition (see later), nicotine was delivered at 30,60 , or $90 \mu \mathrm{g} / \mathrm{kg} /$ infusion; unit dose was calculated from the base form of nicotine. All nicotine infusions were delivered in less than $1 \mathrm{~s}$ at a volume of $0.1 \mathrm{ml} / \mathrm{kg} /$ infusion. Mecamylamine was injected subcutaneously (s.c.) at a dose of $1 \mathrm{mg} / \mathrm{kg}$. This dose was based on preliminary data and previous studies in which higher mecamylamine doses do not have any additional effect on nicotine self-administration (eg Mansbach et al, 2000).

\section{Procedure}

A schematic representation of experimental procedures, dose conditions, and the number of subjects in each condition are presented in Table 1.

Lever training: Rats were autoshaped to press two levers for sucrose pellets as described by Palmatier et al (2006). Rats meeting a criterion of 25 responses during autoshaping sessions were tested for side-preference; the remaining rats were hand shaped to press both levers before preference testing. Preference tests were conducted in extinction (responding was not reinforced), both levers were extended simultaneously and the distribution of responses was used to determine unconditioned side preferences.

After lever training, all rats were randomly assigned to one of four groups (2-Lever, NIC + VS, NIC-Only, or VSOnly) and one of three dose conditions $(30,60$, or $90 \mu \mathrm{g} / \mathrm{kg} /$ infusion). Left $v s$ right lever preferences were counterbalanced as much as allowed by the samples and overall preference ratios for preferred $v s$ non-preferred levers were matched across groups and doses. Subsequently, each rat was randomly assigned an 'active' and 'inactive' lever or an 'infusion' and 'VS' lever with the constraints that left $v s$ right was counterbalanced and preference ratios were matched across lever assignments. Preference ratios in each group and dose condition were not statistically different, $\mathrm{F}<1$.

Surgery: Rats were implanted with chronic indwelling jugular catheters after lever training. Catheters were constructed as described previously (Donny et al, 1998). Following surgery, catheters were flushed daily by infusing $0.1 \mathrm{ml}$ heparinized sterile saline and the antibiotic ticarcillin 
Table I Schematic Representation of Experimental Procedures

Training phase

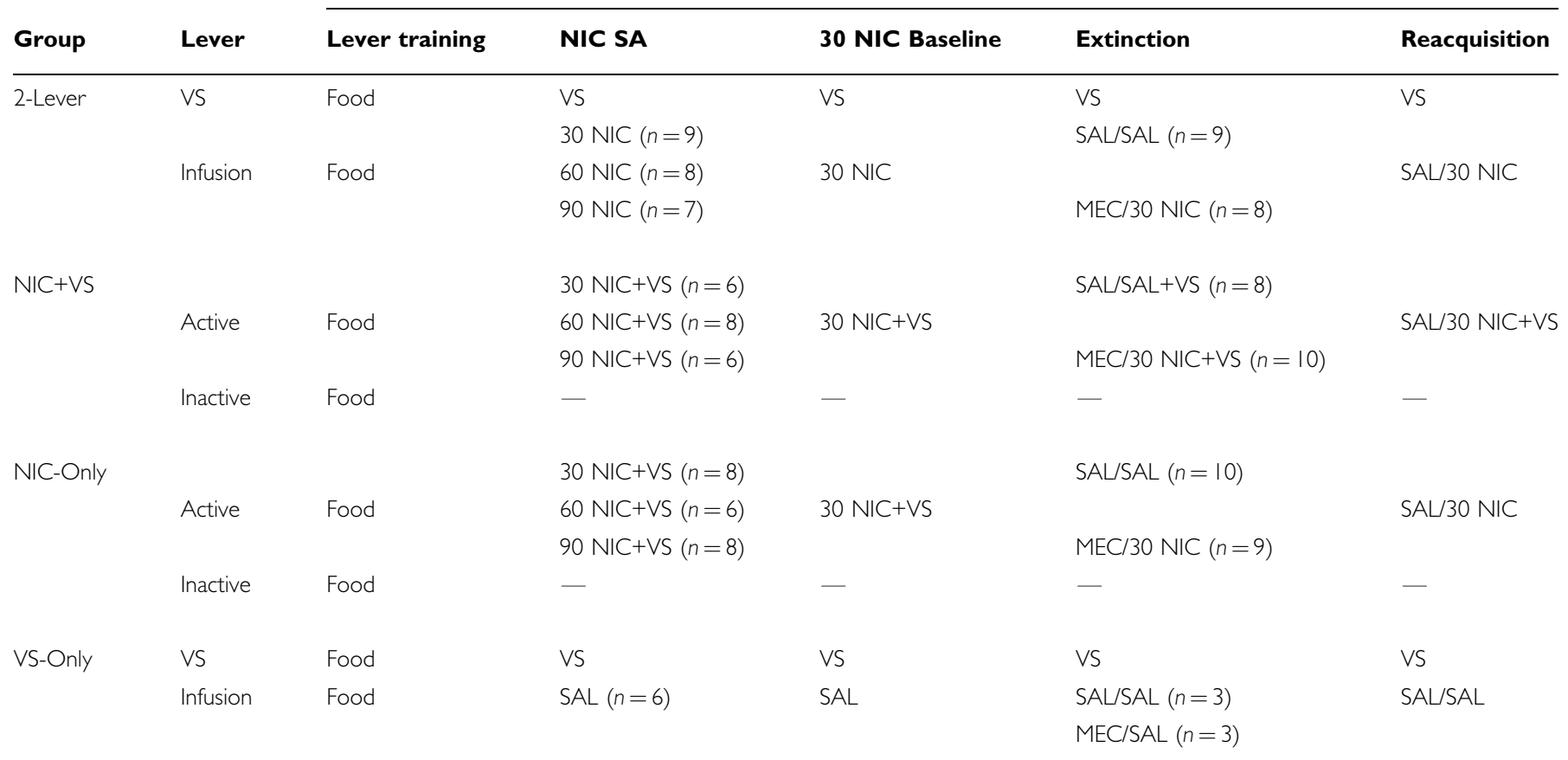

NIC, /NIC: nicotine infusions available during session; SAL, /SAL: vehicle infusions available during session; VS: visual stimulus; 30, 60, 90: unit nicotine dose ( $\mu \mathrm{g} / \mathrm{kg} /$ infusion); MECI, SAL/: mecamylamine or vehicle pretreatment I 5 min before session; $(n=x)$ : number of subjects included in analyses for NIC SA and BaselineExtinction-Reacquisition phases, chloral hydrate tests determined catheter patency after NIC SA and Reacquisition phases.

plus clavulanate to reduce post surgical infections. See Donny et al (1998) for other details about surgical procedures.

Acquisition (Sessions 1-22): Self-administration testing began 7-10 days after surgery and was carried out in 1-h sessions 5 days per week unless otherwise noted. Rats were placed in the experimental chambers and connected to the drug-delivery system. Illumination of the house light signaled the start of the session. For 2-Lever groups, pressing the infusion lever resulted in delivery of the assigned unit dose of nicotine; subsequent infusions were not available until a $1 \mathrm{~min}$ time-out period had expired. Pressing the VS lever resulted in 1-s illumination of the cue light followed by 60 -s deactivation of the house light; pressing the VS-lever had no subsequent programed consequence during this period. For the VS-Only group, training contingencies were identical, however, pressing the infusion lever resulted in a $0.1 \mathrm{ml} / \mathrm{kg}$ infusion of $0.9 \%$ saline. For NIC + VS groups, pressing the active lever delivered an infusion of the assigned unit nicotine dose and the VS. For NIC-Only groups, pressing the active lever resulted only in infusion of the assigned nicotine dose. For these latter two groups, pressing the other (ie inactive) lever had no scheduled consequences. Reinforcement schedules were fixed-ratio 1 (FR1) during sessions 1-12, and FR2 during all remaining sessions. For rats with two active levers (2Lever and VS-Only), the schedule of reinforcement was always equivalent on both levers and similar to rats with one active lever (NIC+VS and NIC-Only, see Palmatier et al, 2006 for more details).
$30 \mu \mathrm{g} / \mathrm{kg}$ baseline (Sessions 23-28): All remaining test sessions were conducted on consecutive days, 7 days per week. During the $30 \mu \mathrm{g} / \mathrm{kg}$ baseline sessions, all groups with access to nicotine (2-Lever, NIC + VS, and NIC-Only) were switched to the $30 \mu \mathrm{g} / \mathrm{kg}$ unit infusion dose. Baseline sessions continued until stability $(\leqslant 30 \%$ variance in reinforcers earned for three consecutive sessions) and similarity (faded doses not significantly different from $30 \mu \mathrm{g} / \mathrm{kg}$ rats over three consecutive sessions) criteria were met. For rats originally assigned to the $30 \mu \mathrm{g} / \mathrm{kg}$ unit dose and for the VS-Only group there were no procedural changes during this phase.

Mecamylamine/saline extinction (Sessions 29-35): Rats in each group were assigned to one of two extinction conditions (MEC-EXT or SAL-EXT) with the constraint that previous unit dose assignment $(30,60$, or $90 \mu \mathrm{g} / \mathrm{kg})$ and responding during the last three baseline sessions were matched across conditions. Rats in the SAL-EXT condition received s.c. injections of saline $15 \mathrm{~min}$ before each session. For these rats, $0.9 \%$ saline vehicle infusions replaced nicotine for meeting the FR2 schedule requirement on the infusion or active lever. Rats in the MEC-EXT condition received s.c. injections of mecamylamine $(1 \mathrm{mg} / \mathrm{kg})$ before each session. For 2-Lever, NIC + VS and NIC-Only rats in the MEC-EXT condition, nicotine infusions were delivered for meeting the response requirement on the infusion/active lever, VS-Only controls continued to receive access to saline infusions on the inactive lever.

Reacquisition (Sessions 36-40): To verify that decreases in responding during extinction were attributable to experi- 
mental manipulations, rats in both extinction conditions were allowed to re-acquire nicotine self-administration. All parameters were identical to the $30-\mu \mathrm{g} / \mathrm{kg}$ baseline phase, except that s.c. saline injections were given $15 \mathrm{~min}$ before each session.

Data analyses: Analyses of response rates were similar to Palmatier et al (2006). Briefly, nicotine seeking was defined as responding on the infusion- or active-lever in the 2-Lever and NIC-Only groups, respectively. VS seeking was defined as responding on the VS-lever in the 2-Lever and VS-Only groups. Nicotine seeking and VS seeking were analyzed independently. Active-lever responding for the NIC+VS group was included in both sets of analyses. Omnibus 3-way ANOVAs for VS-seeking and nicotine-seeking measures included Dose and Group as between subjects factors, and Session as the within subjects factor. Follow-up 2-way ANOVAs contrasted Group within each Dose condition because only one group of rats served as a vehicle baseline for VS seeking (VS-Only group, $n=6$ ). Dunnett's Multiple Comparisons test was used to evaluate significant main effects of Group; responding for multiple reinforcers (ie NIC + VS and 2-Lever groups) was compared to responding from the appropriate control condition (NIC-Only or VSOnly). An a priori alpha criterion was set at $p \leqslant 0.05$ for all comparisons; multiple $p$ values are reported as ' $p$ s'.

\section{RESULTS}

\section{Effect of Nicotine Dose}

Figure 1 illustrates average active-lever responding during each FR1 and FR2 testing session for each group; each nicotine dose $(30,60$, or $90 \mu \mathrm{g} / \mathrm{kg} /$ infusion $)$ condition is displayed in a separate panel (a, b, or c, respectively). Results from the omnibus ANOVAs for nicotine- and VSlever responding during FR1 and FR2 sessions are presented in Table 2.

$30 \mu \mathrm{g} / \mathrm{kg}$ nicotine: When rats had concurrent access to $30 \mu \mathrm{g} / \mathrm{kg}$ nicotine infusions and the VS (2-Lever group), infusion-lever responding reflected the primary reinforcing effect of nicotine - it was similar to NIC-Only controls during FR1 and FR2 testing sessions, $p s \geqslant 0.99$. In contrast, responding for the VS was potently enhanced relative to controls that responded for VS alone on the active lever (VS-Only group), $p s \leqslant 0.02$. Notably, co-presentation of the VS and $30 \mu \mathrm{g} / \mathrm{kg}$ nicotine infusions (NIC + VS group) also potently enhanced responding relative to NIC-Only and VSOnly control groups, $p s \leqslant 0.04$. This difference replicates previous studies from our laboratory (Chaudhri et al, 2006a), although whether it reflects an increase in the motivational value of nicotine or VS is unclear without information provided by the 2 -Lever group.

$60 \mu \mathrm{g} / \mathrm{kg}$ nicotine: This dose condition replicates previous findings (Palmatier et al, 2006). For the 2-Lever group, infusion-lever responding occurred at rates expected by the primary reinforcing effects of nicotine (ie comparable to NIC-Only group), $p s \geqslant 0.34$, and VS-lever responding was increased relative to VS-Only controls, $p=0.02$ (FR2), by the reinforcement-enhancing effects of nicotine. For the NIC + VS group, active-lever responding was higher than VS-Only controls during the FR2 phase, $p=0.03$. Although there was a main effect of Group for infusion-lever
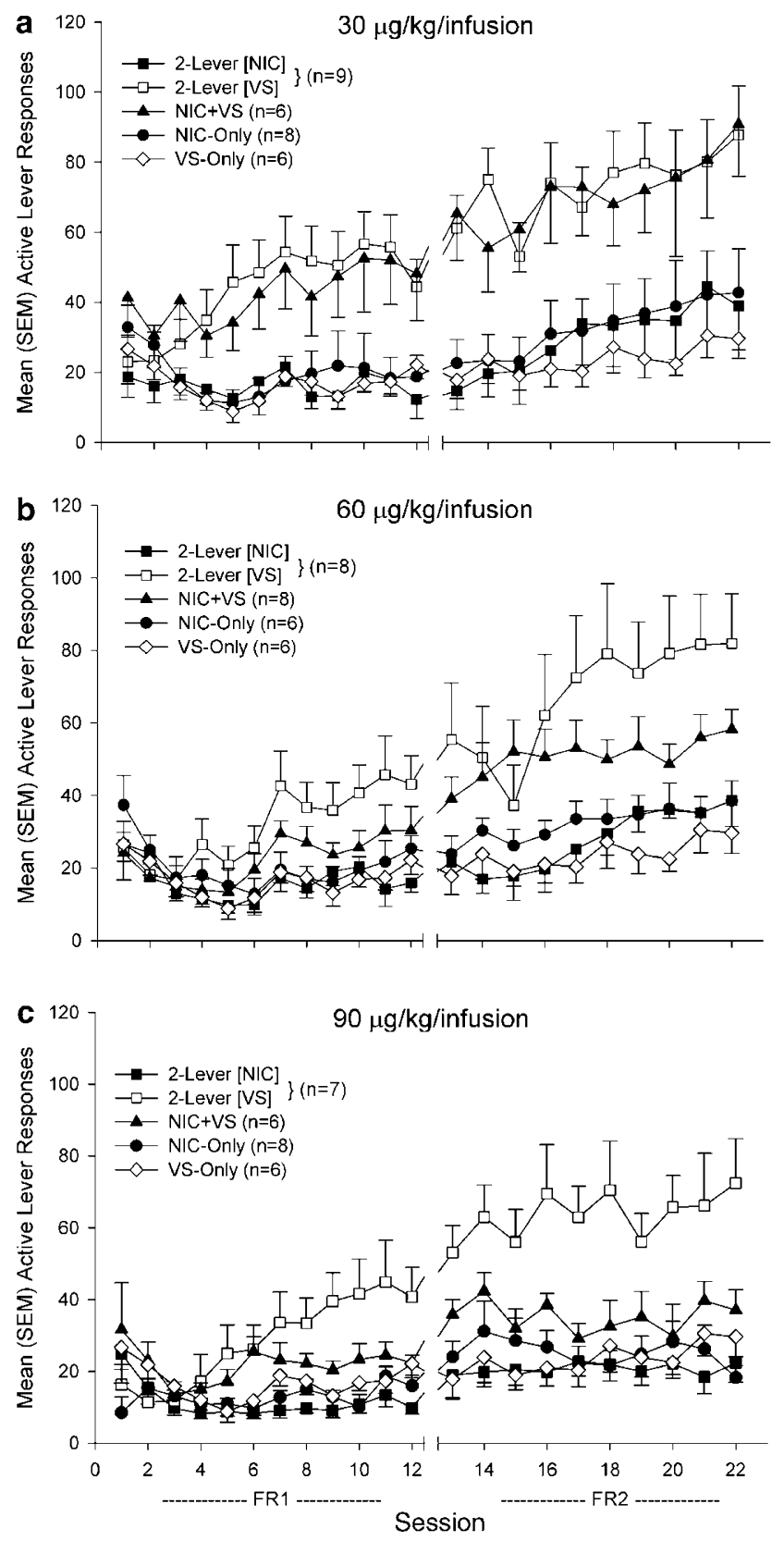

Figure I Mean $( \pm$ I SEM) active lever responding for rats in the NICOnly, NIC + VS, and 2-Lever groups with access to 30 (a), 60 (b), or $90 \mu \mathrm{g} /$ $\mathrm{kg}$ (c) nicotine infusions. For the 2-Lever group, responding on the infusion lever $(\boldsymbol{\square})$ is depicted separately from responding on the VS lever $(\square)$. The VS-Only group ( $0 \mu \mathrm{g} / \mathrm{kg} /$ infusion $)$ is depicted in each panel for comparative purposes. When nicotine and VS were contingent upon a single response (NIC + VS groups) higher unit doses decreased response rate. In contrast, when nicotine and VS were contingent upon separate responses (2-Lever groups), higher unit doses still potently increased responding for VS.

responding during FR1 sessions (see Table 2), neither the NIC + VS nor 2-Lever groups differed from NIC-Only controls, $p s \geqslant 0.08$. For 2 -Lever and NIC + VS groups, the enhancing effect of nicotine seemed to emerge over sessions. The significant Group effect during FR2, but not FR1 sessions, and significant Group $\times$ Session interactions in each phase (VS Presentations, Table 2) confirm this conclusion. Relative to VS-Only controls, the 2-Lever group 
responded at higher rates for the VS on sessions 19-22, $p s \leqslant 0.05$ (corrected, one-tailed), and the NIC + VS group responded at higher rates on sessions $15-17$ and 20 , $p s \leqslant 0.05$ (corrected, one-tailed).

$90 \mu \mathrm{g} / \mathrm{kg}$ nicotine: Concurrent access to $90 \mu \mathrm{g} / \mathrm{kg}$ nicotine and the VS (2-Lever group) was analogous to other dose conditions; infusion-lever responding occurred at rates comparable to the NIC-Only group, $p s \geqslant 0.34$, and VS-lever responding was amplified by the reinforcement-enhancing effects of nicotine, $p=0.001$ (relative to VS-Only, FR2). For the NIC + VS group, co-presentation of the VS and $90 \mu \mathrm{g} / \mathrm{kg}$ nicotine infusions suppressed active-lever responding. In this group, active-lever responding was comparable to VSOnly controls, $p=0.4$ (FR2), but significantly higher than NIC-Only rats, $p s \leqslant 0.002$ (FR1 and FR2). At this unit dose $(90 \mu \mathrm{g} / \mathrm{kg})$, the reinforcement-enhancing effects of nicotine emerged over sessions in the 2-Lever group. There was a significant Group effect during FR2, but not FR1 sessions, and significant Group $\times$ Session interaction during FR1 sessions (VS Presentations, Table 2). Follow-ups confirmed that relative to VS-Only controls, the 2-Lever group responded at higher rates on session $9, p=0.05$ (corrected,

Table 2 Omnibus ANOVA for Response-Rates during FRI and FR2 Sessions

\begin{tabular}{|c|c|c|c|c|c|c|}
\hline \multirow{2}{*}{$\begin{array}{l}\text { Training phase } \\
\text { Source }\end{array}$} & \multicolumn{3}{|c|}{ Infusion lever ${ }^{\mathbf{a}}$} & \multicolumn{3}{|c|}{ VS lever ${ }^{b}$} \\
\hline & df & $\mathbf{F}$ & Sig. & df & $\mathbf{F}$ & Sig. \\
\hline \multicolumn{7}{|l|}{ FRI (sessions 1-12) } \\
\hline Session & 11 & $\mid 4.84$ & $0.00 *$ & II & 29.88 & $0.00 *$ \\
\hline Session* dose & 22 & 1.32 & 0.15 & 22 & 1.32 & 0.15 \\
\hline Session* group & 22 & 6.50 & $0.00 *$ & II & 4.46 & $0.00 *$ \\
\hline Session* dose* group & 44 & 1.17 & 0.22 & 22 & 0.58 & 0.94 \\
\hline Error (session) & 627 & & & 473 & & \\
\hline Dose & 2 & 7.36 & $0.01 *$ & 2 & 6.41 & $0.01 *$ \\
\hline Group & 2 & 26.79 & $0.00 *$ & । & 2.25 & 0.14 \\
\hline Dose* group & 4 & 4.03 & $0.01 *$ & 2 & 0.23 & 0.80 \\
\hline Error & 57 & & & 43 & & \\
\hline \multicolumn{7}{|l|}{ FR2 (sessions 13-22) } \\
\hline Session & 9 & 5.64 & $0.00 *$ & 9 & 4.99 & $0.00 *$ \\
\hline Session* dose & 18 & 2.90 & $0.00 *$ & 18 & 1.19 & 0.26 \\
\hline Session* group & 18 & 1.46 & 0.10 & 9 & 2.48 & $0.01 *$ \\
\hline Session* Dose* group & 36 & 0.69 & 0.92 & 18 & 1.13 & 0.32 \\
\hline Error (session) & 513 & & & 387 & & \\
\hline Dose & 2 & 4.36 & $0.02 *$ & 2 & 3.95 & $0.03 *$ \\
\hline Group & 2 & 29.99 & $0.00 *$ & । & 6.67 & $0.01 *$ \\
\hline Dose* group & 4 & 2.67 & $0.04 *$ & 2 & 0.70 & 0.50 \\
\hline Error & 57 & & & 43 & & \\
\hline
\end{tabular}

$F R=$ fixed ratio schedule of reinforcement.

*Indicates significant main effect or interaction according to a priori criterion, $p \leqslant 0.05$.

ancludes infusion (active)-lever responding for 2-Lever, NIC+VS, and NIC-Only groups, 30-90 $\mu \mathrm{g} / \mathrm{kg} /$ infusion doses.

IIncludes VS (active)-lever responding for 2-Lever, NIC+VS, and VS-Only groups, $0-90 \mu \mathrm{g} / \mathrm{kg} /$ infusion doses. one-tailed) and throughout FR2 sessions (see previous). NIC + VS groups were not included in these follow-ups because active-lever responding never differed from VSOnly controls (Table 3 ).

Dose-response analyses: Figure 2a illustrates the doseresponse curves generated from average responding during the last three FR2 testing sessions. The pattern confirms that high unit doses of nicotine inhibit responding for VS when both reinforcers are contingent on a single response (NIC + VS group). In contrast, when nicotine and VS are contingent on separate responses, nicotine intake depends on the drug's primary reinforcing effect (similar to NICOnly controls), but the reinforcement-enhancing effect is maximal in all dose conditions. These conclusions were

Table 3 Follow-Up Two-Way ANOVAs for Response Rates Within Each Nicotine Dose Condition

\begin{tabular}{|c|c|c|c|c|c|c|c|c|}
\hline \multirow[b]{2}{*}{ Dose } & \multirow[b]{2}{*}{ Phase } & \multirow[b]{2}{*}{ Source } & \multicolumn{3}{|c|}{ Infusion lever $^{\mathbf{a}}$} & \multicolumn{3}{|c|}{ VS lever ${ }^{\mathbf{b}}$} \\
\hline & & & df & $\mathbf{F}$ & Sig. & df & $\mathbf{F}$ & Sig. \\
\hline \multirow[t]{10}{*}{$30 \mu \mathrm{g} / \mathrm{kg} / \mathrm{inf}$} & FRI & Session & 11 & 4.24 & $0.00 *$ & 11 & | 4.23 & $0.00 *$ \\
\hline & & Session* group & 22 & 3.37 & $0.00 *$ & 22 & 3.26 & $0.00 *$ \\
\hline & & Error (session) & 220 & & & 198 & & \\
\hline & & Group & 2 & 12.06 & $0.00 *$ & 2 & 4.88 & $0.02 *$ \\
\hline & & Error & 20 & & & 18 & & \\
\hline & FR2 & Session & 9 & 6.21 & $0.00 *$ & 9 & 2.82 & $0.01 *$ \\
\hline & & Session* group & 18 & 0.75 & 0.76 & 18 & 0.70 & 0.81 \\
\hline & & Error (session) & 180 & & & 162 & & \\
\hline & & Group & 2 & 11.21 & $0.01 *$ & 2 & 8.20 & $0.01 *$ \\
\hline & & Error & 20 & & & 18 & & \\
\hline \multirow[t]{10}{*}{$60 \mu g / k g / i n f$} & FRI & Session & 11 & 8.24 & $0.00 *$ & 11 & 14.13 & $0.00 *$ \\
\hline & & Session* group & 22 & 2.66 & $0.00 *$ & 22 & 2.13 & $0.01 *$ \\
\hline & & Error (session) & 209 & & & 209 & & \\
\hline & & Group & 2 & 5.70 & $0.01 *$ & 2 & 3.24 & 0.06 \\
\hline & & Error & 19 & & & 19 & & \\
\hline & FR2 & Session & 9 & 3.55 & $0.00 *$ & 9 & 5.30 & $0.00 *$ \\
\hline & & Session* group & 18 & 1.06 & 0.40 & 18 & 1.98 & $0.01 *$ \\
\hline & & Error (session) & 171 & & & 171 & & \\
\hline & & Group & 2 & 15.12 & $0.00 *$ & 2 & 3.84 & $0.04 *$ \\
\hline & & Error & 19 & & & 19 & & \\
\hline \multirow[t]{10}{*}{$90 \mu \mathrm{g} / \mathrm{kg} / \mathrm{inf}$} & FRI & Session & II & 8.34 & $0.00 *$ & 11 & | $1.7 \mid$ & $0.00 *$ \\
\hline & & Session* group & 22 & 2.40 & $0.00 *$ & 22 & 3.51 & $0.00 *$ \\
\hline & & Error (session) & 198 & & & 176 & & \\
\hline & & Group & 2 & 22.01 & $0.00 *$ & 2 & 3.01 & 0.08 \\
\hline & & Error & 18 & & & 16 & & \\
\hline & FR2 & Session & 9 & 0.72 & 0.69 & 9 & 1.71 & 0.09 \\
\hline & & Session* Group & 18 & 1.17 & 0.30 & 18 & 0.84 & 0.65 \\
\hline & & Error (session) & 162 & & & 144 & & \\
\hline & & Group & 2 & 13.22 & $0.00 *$ & 2 & 10.95 & $0.00 *$ \\
\hline & & Error & 18 & & & & & \\
\hline
\end{tabular}

$F R=$ fixed ratio schedule of reinforcement.

*Indicates significant main effect or interaction according to a priori criterion, $p \leqslant 0.05$.

${ }^{a}$ Contrasts 2-Lever, NIC+VS, and NIC-Only groups.

${ }^{b}$ Contrasts 2-Lever, NIC+VS and VS-Only $(0 \mu \mathrm{g} / \mathrm{kg} /$ infusion $)$ groups. 

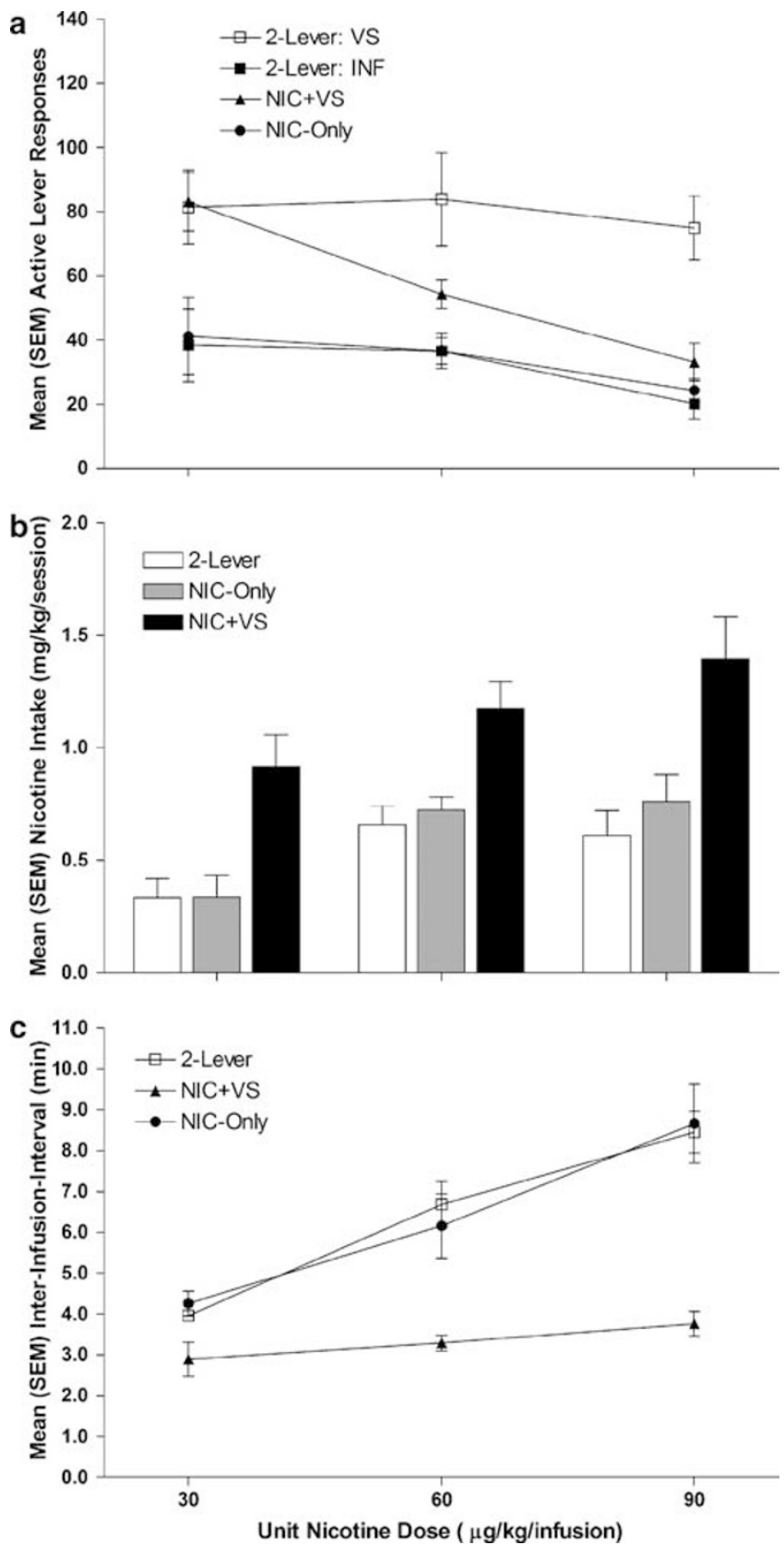

Figure 2 Mean ( \pm I SEM) reinforcers earned (top panel), nicotine intake (center panel), and inter-infusion interval (bottom panel) as a function of unit infusion dose $(\mu \mathrm{g} / \mathrm{kg} /$ infusion) for rats with access to nicotine. Data points represent averages from the last 3 days of SA Testing (sessions 2022). When nicotine and VS were contingent upon a single response (NIC +VS groups) higher unit doses decreased the number of reinforcers earned but increased nicotine intake and did not change inter-infusion intervals. In contrast, when only nicotine was self-administered (NIC-Only groups) or when drug infusions and VS were contingent upon separate responses (2-Lever groups), nicotine intake was regulated and maximally increased responding for VS (2-Lever groups). Higher unit doses did not increase intake and inter-infusion intervals increased as a function of unit dose.

confirmed by separate two-way ANOVAs on VS- and infusion-lever responding with significant main effects of Group and Dose, $p s<0.01$, and significant Group $\times$ Dose interactions, $p s \leqslant 0.034$. To further investigate, the effect of manipulating nicotine dose on the primary reinforcing and reinforcement-enhancing effects of nicotine, average drugintake across the last three FR2 test sessions (mg/kg/ session) was calculated and expressed as a function of unit dose in Figure 2b. Co-presentation of nicotine and VS results in higher total nicotine intake relative to rats making independent responses for nicotine and VS. When both reinforcers were contingent on a single operant response (NIC + VS group), the reinforcement-enhancing effect of nicotine may inflate drug intake by increasing the incentive value of the non-pharmacological stimuli that are presented in conjunction with drug infusions. Multivariate ANOVA revealed significant main effects of Unit Dose and Group and a Significant Unit Dose $\times$ Group interaction, $p s \leqslant 0.02$.

The relationship between unit nicotine dose and total nicotine intake suggests that rats with independent control of nicotine infusions (2-Lever and NIC-Only groups) regulated their drug intake. In contrast, when both reinforcers were delivered for making a single response, nicotine intake increased monotonically with unit dose. Regulating nicotine intake should be expressed as longer inter-reinforcer (infusion) -intervals (IIIs) for rats with access to higher unit doses. To investigate this possibility, we calculated IIIs for the three groups with access to nicotine over the 3 days that were used to generate doseresponse curves (sessions 20-22). Average intervals from rats that received a minimum of three infusions per session are illustrated in Figure 2c. For rats with independent control over nicotine infusions, IIIs increased as unit nicotine dose increased. In contrast, for rats in the NIC + VS group, III did not vary as a function of unit nicotine dose. These conclusions were confirmed by omnibus ANOVA with a significant Group $\times$ Unit Dose interaction, $p=0.02$, and follow-ups contrasting dose in each group; IIIs did not differ across doses in the NIC + VS group, $p s \geqslant 0.11$, for 2-Lever and NIC-Only groups, rats receiving 60 and $90 \mu \mathrm{g} / \mathrm{kg} /$ infusion had significantly higher IIIs than the $30 \mu \mathrm{g} / \mathrm{kg} /$ infusion conditions, $p s \leqslant 0.01$. Notably, latency to receive the first infusion (data not shown) did not vary systematically as a function of Group or Dose, $p s \geqslant 0.29$.

Inactive levers: Some of the groups in the present studies had inactive levers (NIC + VS and NIC-Only) or levers that only delivered saline infusions (VS-Only). Therefore, we determined whether response rates on the inactive or saline associated lever differed from response rates on the lever associated with reinforcement. The VS-Only group responded at higher rates on the VS-lever relative to the infusion lever. This conclusion was supported by significant main effects of Lever and Session during FR1 and FR2 sessions, $p s \leqslant 0.001$. For the NIC + VS and NIC-Only groups, rats responded at higher rates on active levers, relative to inactive levers, regardless of nicotine dose. This was confirmed by three-way ANOVAs with significant main effects of Lever and Session during both FR1 and FR2 testing, $p s<0.001$. There was also a significant Lever $\times$ Dose interaction for the NIC+VS group during FR2 sessions, $p=0.011$.

\section{Mecamylamine/Saline Extinction}

$30 \mu \mathrm{g} / \mathrm{kg}$ unit dose baseline: Rats that received access to 60 or $90 \mu \mathrm{g} / \mathrm{kg}$ nicotine infusions during self-administration 
testing were switched to the $30 \mu \mathrm{g} / \mathrm{kg}$ unit dose. Under these conditions, self-administration testing continued until rats in each preceding dose condition $(30,60$, or $90 \mu \mathrm{g} / \mathrm{kg} /$ infusion) responded at similar rates for a minimum of 3 consecutive days. Stable performance was evident after 6 days of testing with the $30 \mu \mathrm{g} / \mathrm{kg}$ infusion dose (Fs $<1$ ).

Extinction and reacquisition: Rats from each group were randomly assigned to one of two extinction treatments (SAL-EXT or MEC-EXT) with the constraints that preceding dose condition and responding during the baseline phase were matched. Figure 3 illustrates active-lever responding for each group during the four test conditions of interest: baseline (E0, last three sessions of baseline phase), acute extinction (E1, first day of MEC challenge/saline substitution), chronic extinction (E7, 7th day of MEC challenge/ saline substitution), and re-acquisition (R4, 4th day retraining with nicotine). The ANOVAs included Test as a within subjects factor, Group (2-Lever, NIC + VS, etc.) and Extinction Treatment (MEC-EXT and SAL-EXT) were between subjects factors. Follow-ups exploring GroupTest interactions used paired-samples $t$-tests (responding from each test compared with the subsequent test) with Bonferroni's alpha correction (eg $\alpha=0.05 / 3$ comparisons). Mecamylamine and saline extinction treatments did not differ in their impact on VS- and infusion-lever responding, Fs $\leqslant 0.98$. Because of the small sample size in the VS-Only group $(n=6)$ responding in the two extinction treatment conditions was pooled for follow-up comparisons. Figure 4 illustrates average active-lever responding throughout each phase; these data were included for visual inspection and were not included in the analyses.

The reinforcement-enhancing effect of nicotine depends on acute action of nicotine at nAChRs. For the 2-Lever and $\mathrm{NIC}+\mathrm{VS}$ groups, VS-lever responding declined as the result of acute extinction treatment (extinction day 1 ) and did not

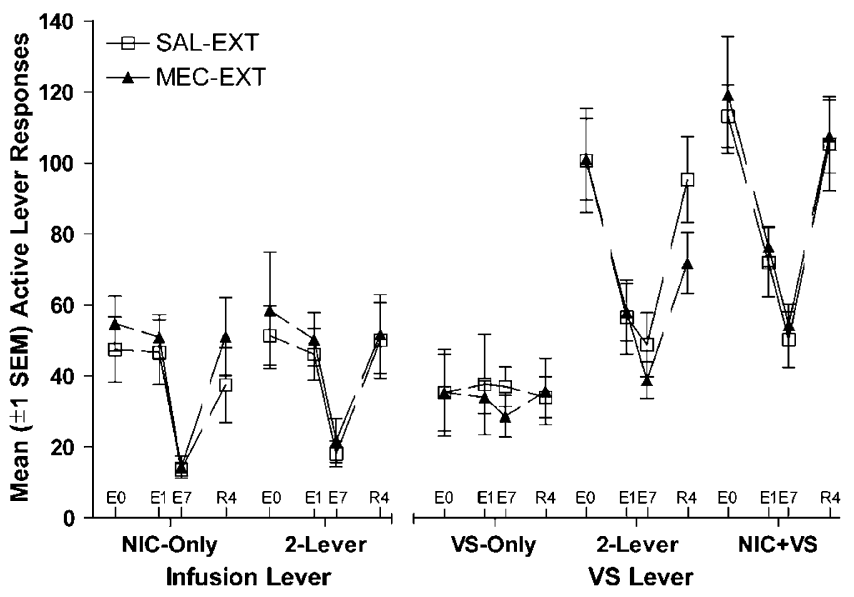

Figure 3 Mean $( \pm$ I SEM) active lever responses for 2-Lever, NIC + VS, NIC-Only, and VS-Only groups during the baseline (EO, last three sessions of $30 \mu \mathrm{g} / \mathrm{kg} /$ infusion baseline phase), acute extinction (EI, first day of MEC challenge/saline substitution), chronic extinction (E7, 7th day of MEC challenge/saline substitution), and re-acquisition (R4, 4th day re-training with nicotine). The mecamylamine pretreatment and saline substitution conditions did not differ from one another in any group. The reinforcement-enhancing effects of nicotine were dependent on the acute action of nicotine at $\mathrm{nAChRs}$ (right panel). In contrast, the primary reinforcing effects of nicotine required a new association between the lever and attenuated nAChR activity (ie extinction, left panel). recover until reacquisition. Chronic extinction treatment did not have any additional impact on the reinforcementenhancing effect of nicotine beyond that produced on the first extinction day (2-Lever group). However, when these enhancing effects were superimposed on primary reinforcement (NIC + VS group), chronic treatment resulted in some additional decrement. These conclusions were confirmed by significant main effects of Group and Test, and a significant Group $\times$ Test interaction, $p s \leqslant 0.004$. For $\mathrm{NIC}+\mathrm{VS}$ and 2-Lever groups, follow-ups revealed a significant decrease in responding after acute extinction (E0 vs E1) and a significant increase after re-acquisition (E7 vs R4), $p s<0.001$. For the 2-Lever group, responding did not change during the extinction phase (E1 vs E7), $p=0.21$; however for the NIC+VS group, active-lever responding declined during extinction, $p=0.02$. For both groups, VSlever responding was comparable to VS-Only controls during the last extinction session (E7), $p s \geqslant 0.15$.

The primary reinforcing effect of nicotine depends on both nicotinic-cholinergic function and an expectation of the reinforcing outcome. For the 2-Lever and NIC-Only groups, infusion-lever responding was not affected by acute extinction treatment. In these groups, nicotine seeking declined progressively across the extinction phase and was re-acquired when the effects of nicotine were re-introduced. These conclusions were confirmed by significant main effects of Group and Test and a significant Group $\times$ Test interaction, $p s \leqslant 0.002$. Follow-ups confirmed acute extinction (E0 vs E1) did not attenuate responding at the drug lever in 2 -Lever and NIC-Only groups, $p s \geqslant 0.11$. However, responding decreased after chronic testing (E1 vs E7), $p s \leqslant 0.003$, and increased after re-acquisition (E7 vs R4), $p s \leqslant 0.001$.

The results suggested that the reinforcement-enhancing effects of nicotine were immediately abolished by extinction treatment, whereas primary reinforcement decremented over successive treatments (Figure 3). Additional analyses examined the pattern of responding across $10 \mathrm{~min}$ intervals for the last $30 \mu \mathrm{g} / \mathrm{kg}$ baseline session and the first extinction session (Figure 4) in order to determine whether any enhancing effect of nicotine was retained during the first extinction session. Omnibus ANOVAs revealed that no main effect or interaction involving extinction condition (MEC-EXT vs SAL-EXT) was significant, $p s \geqslant 0.31$, so data from these conditions were pooled. During the last baseline session and first extinction session, the 2-Lever group demonstrates a pattern of responding that is consistent with the pattern seen across extinction sessions. The VS immediately loses value as the result of extinction treatment (Figure 4b), whereas nicotine seeking does not change (Figure $4 \mathrm{a}$ and $\mathrm{c}$ ). This finding was confirmed by two-way within groups (Session $\times$ Interval) ANOVAs. For infusionlever responding, only the main effect of Interval was significant, $p<0.001$ (this pattern was identical for NICOnly group, $p<0.001)$. For VS-lever responding only the main effect of Session was significant, $p=0.001$. The significant main effects and interaction observed for the NIC + VS group (Interval, Session, and Session $\times$ Interval, $p s<0.001)$ suggests that responding was normally maintained during the session by enhanced VS seeking (day 28), but this was decremented during the first session of extinction treatment. 

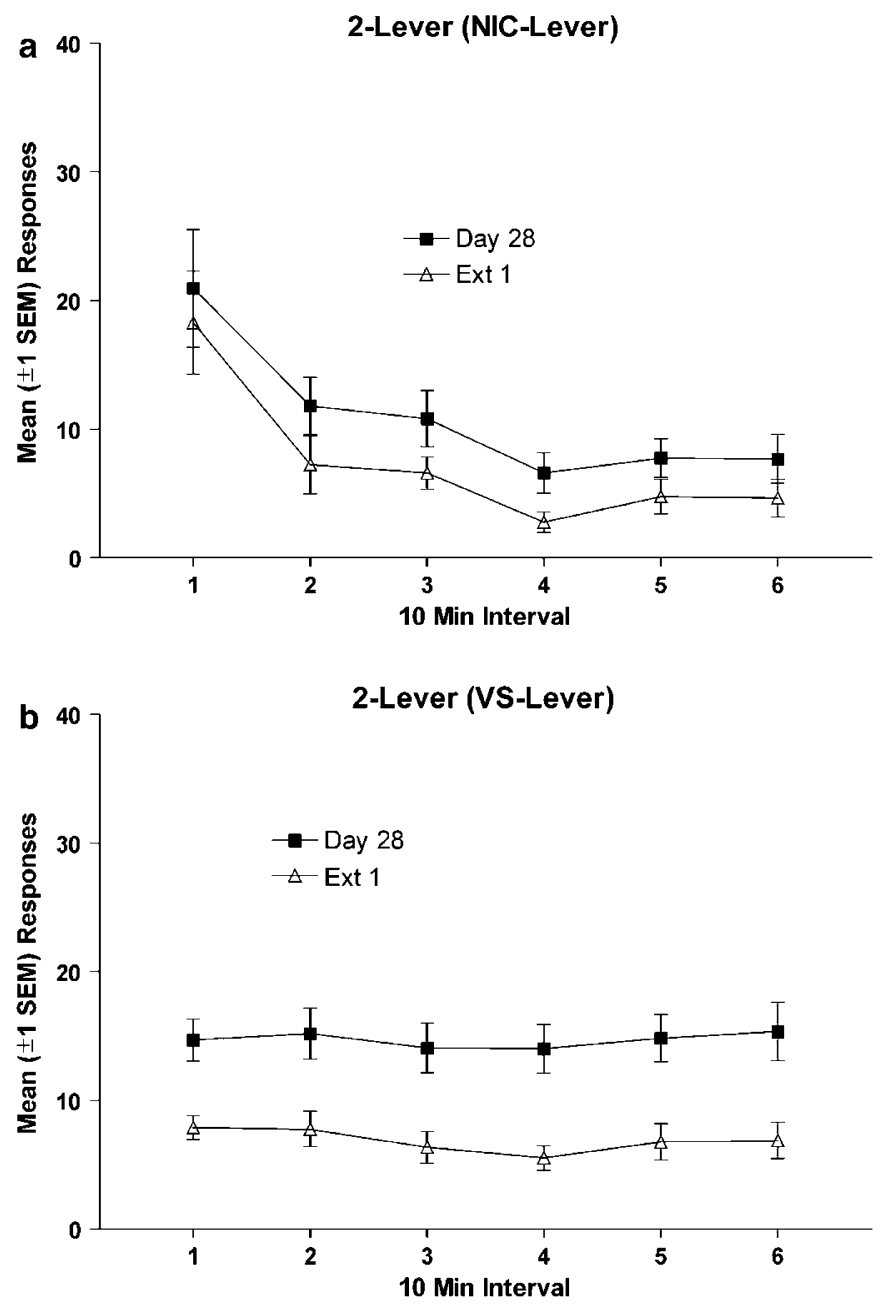

C
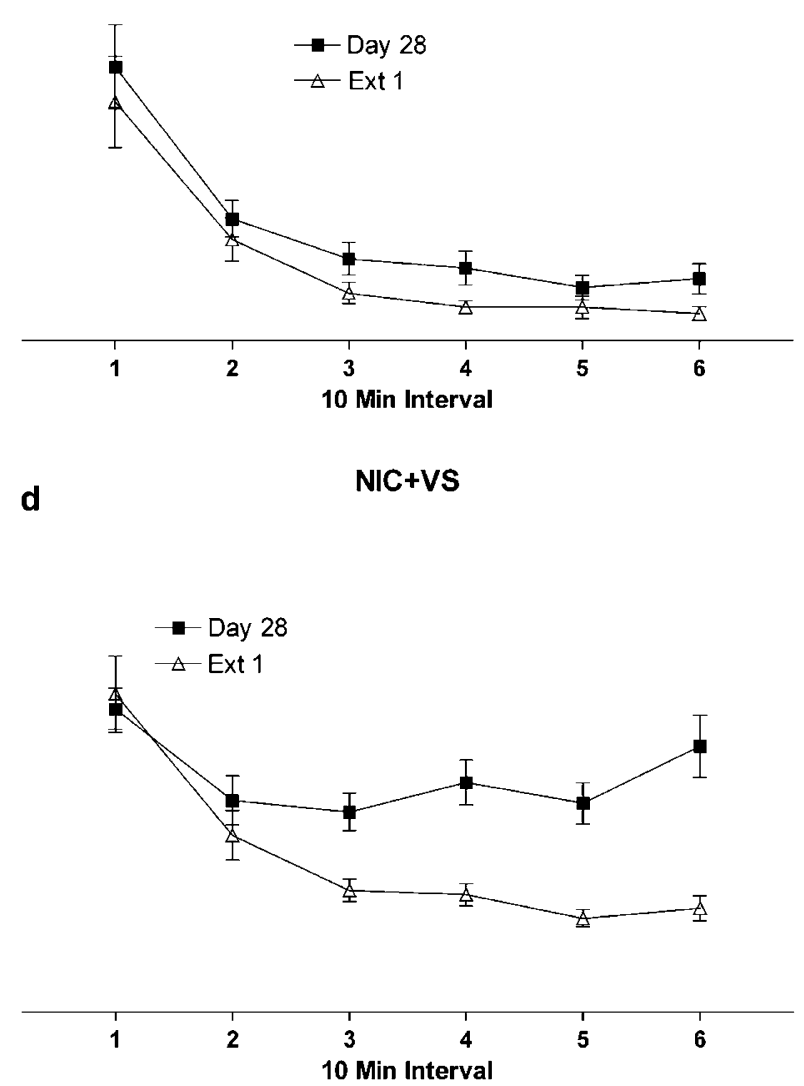

Figure 4 Mean ( \pm I SEM) active lever responses for 2-Lever (a and b), NIC-Only (c), and NIC + VS (d) groups plotted as I0-min intervals from the last $30 \mu \mathrm{g} / \mathrm{kg} /$ infusion baseline session (day 28) and first extinction session (Ext I). The reinforcement-enhancing effect of nicotine was abolished as soon as the effects of nicotine were attenuated (b). Within-session responding for the primary reinforcing effect of nicotine (a and c) supports the conclusion that attenuation of this effect requires new learning. For the NIC + VS group (d), the pattern of responding across the first extinction session may represent new learning about lever-nicotine and/or VS-nicotine contingencies.

\section{DISCUSSION}

Nicotine is an agonist at central nervous system nAChRs and this action is generally considered to be the pharmacological substrate for nicotine-reinforcement (eg Corrigall and Coen, 1989). However, we have recently demonstrated that nicotine has at least two effects on reinforcement (Chaudhri et al, 2006b; Donny et al, 2003): it enhances the incentive value of a reinforcing non-pharmacological stimulus (reinforcement-enhancing effect) and also supports operant responding when no other outcome is contingent on this behavior (primary reinforcing effect, see Chaudhri et al, 2006a for review). These findings raise important questions about the role of nicotinic systems in reinforcement, namely, how do nAChRs mediate each reinforcement-related effect of nicotine? The present studies were designed to examine the contribution of nAChRs to the primary reinforcing and reinforcement-enhancing effects of nicotine using a paradigm that can systematically dissociate the two phenomena (Palmatier et al, 2006). Three new findings emerged that may help to refine our under- standing of nicotine's incentive properties. First, the primary reinforcing effects of nicotine depend on $n A C h R$ activity and an 'expectation' of a reinforcing drug effect. Second, the reinforcement-enhancing effects of nicotine depend on acute pharmacological activity of nAChRs. Third, under standard self-administration training protocols, responding for a non-pharmacological stimulus may inflate total nicotine intake.

Although both the primary reinforcing and reinforcement-enhancing effects of nicotine emerged gradually over self-administration sessions, their reliance on nAChR function differed substantially. The primary reinforcing effects of nicotine were mediated by an interaction between nAChR function and some behavioral/neurobiological process that changed over time (Figure 3 left panel). As mecamylamine challenge and saline substitution produced patterns of responding that were identical over all phases of the study, it is unlikely that a cumulative effect of mecamylamine treatment was responsible for decreasing operant responding. Rats in the 2-Lever and NIC-Only groups that received SAL-EXT treatment had to make a new 
association between the operant response and a change in availability of the drug reinforcer (nicotine). The comparable change in the MEC-EXT group suggests that after mecamylamine challenge the infusion-lever response had to enter into a new association with decreased efficacy of nicotine at nAChRs. This extinction-like effect of mecamylamine is not novel (Donny et al, 1999; Risner and Goldberg, 1983; Shoaib et al, 1997), but in the present study, it is uncontaminated by co-presentation of a stimulus during the acquisition of 'nicotine reinforcement'. In prior studies, decremental responding induced by acute/chronic mecamylamine may represent extinction of response-nicotine associations, extinction of stimulus-nicotine associations, or loss of the reinforcement-enhancing effect (see later). However, in the present study, the 2-Lever and NIC-Only groups isolate nicotine seeking to associations between the operant response and nicotine's effect at nAChRs.

Although the effect of mecamylamine on nicotine reinforcement is clear in the 2-Lever and NIC-Only groups, it is somewhat surprising that decreasing the effect of nicotine at the receptor does not decrease nicotine seeking. Decreased nicotine seeking after acute mecamylamine treatment in previous studies (eg Corrigall and Coen, 1989) suggests that nAChRs mediate the motivation to take nicotine or the drug's incentive value. However, there are presumably two processes guiding responding on the nicotine lever in the present study: the motivation to take nicotine (the drugs incentive value) and the associative history between nicotine-delivery and the operant response. In the present studies, mecamylamine did not change incentive value, but permitted a change in the associative history of the operant response. Incentive value is often attributed to mesocorticolimbic circuits that mediate associations between rewards and stimuli/responses contiguous with those rewards (Balfour et al, 2000; Corrigall et al, 1994; Di Chiara, 2000; Laviolette and van der Kooy, 2004; Picciotto and Corrigall, 2002). The incentive value of nicotine likely depends on induction of long-term changes in synaptic function that are facilitated by, but not dependent on, continued $\mathrm{nAChR}$ activity in these regions. For example, nicotine causes long-term facilitation of glutamate release from prefrontal cortical neurons projecting to the ventral tegmental area (VTA, Mansvelder et al, 2002; Schilström et al, 2003). This facilitation is thought to increase burst firing of VTA cells and increase extracellular dopamine in VTA target regions (eg Gonon, 1988). If facilitation of glutamatergic communication in the VTA is responsible for the nicotine's incentive value, then disrupting of glutamatergic activity in the VTA should immediately decrease primary reinforcement. Notably, Laviolette and van der Kooy (2003) showed that VTA administration of the $\mathrm{N}$-methyl-D-aspartate glutamate receptor antagonist AP-7 decreased nicotine-induced conditioned place preference, perhaps by disrupting the drugs incentive value.

In contrast to the primary reinforcing effects of nicotine, the reinforcement-enhancing effects depend only on the acute action of nicotine at nAChRs (right panel of Figures 3 and $4 \mathrm{~b}$ ). As soon as nicotine was removed (SAL-EXT group) or nicotinic activity attenuated (MEC-EXT group) responding for the VS returned to basal levels (Figure 3). The pattern of VS seeking within the first extinction session generally supports this conclusion; when nicotine- and
VS-seeking are unconfounded by co-presentation (ie 2-Lever group), no enhancing effect is evident during any period of the first extinction session (Figure 4b). This finding contrasts with previous studies demonstrating that nicotine enhances a goal-directed conditional response (Olausson et $a l, 2003)$ and responding with conditioned reinforcement (Olausson et al, 2003, 2004) for up to 14 days after the last drug exposure. An important future direction of the present research will be to determine whether the effects of nicotine on conditional reinforcement (Olausson et al, 2004) differ from its effects on unconditional or sensory reinforcement (Palmatier et al, 2006, present studies). Conditional responses and reinforcers may differ from unconditional reinforcers in a manner that makes them more susceptible to the influence of prior nicotine exposure. For example, prior exposure to low nicotine doses (eg $0.18 \mathrm{mg} / \mathrm{kg}$ for 9 days) may enhance associative learning (Bevins and Palmatier, 2003) and therefore acquisition and expression of conditional responses (Olausson et al, 2003, 2004).

The dose-response relationships obtained for the NIC+ VS and NIC-Only groups (Figure 2) replicate curves we have previously reported for nicotine when drug infusions are co-presented with another reinforcing stimulus (Donny et al, 1999) and when drug infusions are delivered alone (Donny et al, 2003). When nicotine infusions are the only reinforcer available under a fixed ratio schedule, the doseresponse relationship is relatively flat (Figure $2 \mathrm{a}$, Donny et al, 2003). Under 'standard' self-administration protocols (NIC + VS group), unit dose is inversely related to FR response rates (Figure 2a, Donny et al, 1999). Under FR schedules of drug self-administration, the inverse relationship between unit dose and response rate is often attributed to satiety (eg Wise, 1987). Therefore, it is tempting to conclude that in the NIC + VS groups, high unit doses of nicotine would increase responding for VS, except for a satiating effect of nicotine that inhibits responding on a lever associated with drug infusions. However, previous studies of the reinforcement-enhancing effect (Chaudhri et al, 2006c; Liu et al, 2005) invalidate this account. When nicotine infusions are yoked to NIC + VS groups (Chaudhri et al, 2006c) the dose-response relationship does not differ from NIC + VS groups, response rate declines as unit dose increases. This finding argues against satiety-mediated decreases; yoked groups were not responding for drug infusions so drug satiety should not inhibit responding. Responding in NIC+VS groups at higher unit doses probably reflects psychomotor inhibition or some aversive effect of nicotine. However, the relationship between nicotine dose and the reinforcement-enhancing effect, as well as the motivational factors mediating decreased responding under FR schedules, require further experimental investigation.

As finding that nicotine has an unconditioned enhancing effect on reinforcement, we have considered many questions about the nature of the phenomenon and the circumstances under which it is manifest. For example, how is this effect involved in tobacco addiction? The doseresponse findings from the present study may provide an initial answer to this question. Nicotine intake is inflated by sensory reinforcers only when both outcomes are dependent on the same operant. Sensory reinforcers tied to nicotine intake (taste/smell of smoke, etc.) may also inflate 
drug taking in human smokers. For example, smokers rate intravenous nicotine delivery to be much less satisfying than access to denicotinized smoke (Rose et al, 2000) and puffing denicotinized cigarettes has a stronger satiating effect on ad lib smoking than pulsed infusions of nicotine (Rose et al, 2003). More sensitivity to the sensorimotor component of smoking, relative to nicotine intake, probably reflects a combination of conditional value bestowed upon these sensorimotor cues by prior association with nicotine as well as a direct inflation of their incentive value by the reinforcement-enhancing effects of nicotine. However, in traditional single-lever paradigms, the dual reinforcing effects of nicotine make it difficult to determine which variables control responding and must be mitigated by a 'control' group in which both reinforcers are available, selfadministered, and non-competitive. When these technical details are considered, both the concurrent access (2-Lever) and paired stimulus (NIC + VS) paradigms can offer a great deal of insight into the behavioral and biological factors that mediate tobacco addiction.

\section{ACKNOWLEDGEMENTS}

We thank Sheri Booth, Maysa Gharib, Laure Craven, Prema Chaudhri, and Jessica Black for their assistance conducting the studies. All experiments followed the 'Principles of laboratory animal care' (NIH \#85-23, revised 1985) and were approved by the University of Pittsburgh Institutional Animal Care and Use Committee (Assurance \# A3187-01). This research was supported by NIH Grants DA-10464, DA-12655, and DA-19278.

\section{REFERENCES}

Balfour DJ, Wright AE, Benwell ME, Birrell CE (2000). The putative role of extra-synaptic mesolimbic dopamine in the neurobiology of nicotine dependence. Behav Brain Res 113: 73-83.

Bevins RA, Palmatier MI (2003). Effects of nicotine preexposure on the conditioned and unconditioned psychomotor effects of nicotine in rats. Behav Brain Res 143: 65-74.

Caggiula AR, Donny EC, White AR, Chaudhri N, Booth S, Gharib MA et al (2002). Environmental stimuli promote the acquisition of nicotine self-administration in rats. Psychopharmacology 163: 230-237.

Chaudhri N, Caggiula AR, Donny EC, Booth S, Gharib M, Craven L et al (2006b). Self-administered and noncontingent nicotine enhance reinforced operant responding in rats: impact of nicotine dose and reinforcement schedule. Psychopharmacology, doi:10.1007/s00213-006-0522-0.

Chaudhri N, Caggiula AR, Donny EC, Booth S, Gharib M, Craven L et al (2006c). Dissociating the dual-actions of nicotine: primary reinforcement and the reinforcement-enhancing actions of nicotine impact operant responding for a visual stimulus in rats. Psychopharmacology, doi:10.1007/s00213-006-0454-8.

Chaudhri N, Caggiula AR, Donny EC, Palmatier MI, Liu X, Sved AF (2006a). Complex interactions between nicotine and nonpharmacological stimuli reveal multiple roles for nicotine in reinforcement. Psychopharmacology 184: 353-366.

Corrigall WA, Coen KM (1989). Nicotine maintains robust selfadministration in rats on a limited-access schedule. Psychopharmacology 99: 473-478.

Corrigall WA, Coen KM (1994). Nicotine self-administration and locomotor activity are not modified by the 5-HT3 antagonists ICS 205-930 and MDL 72222. Pharmacol Biochem Behav 49: $67-71$.
Corrigall WA, Coen KM, Adamson KL (1994). Self-administered nicotine activates the mesolimbic dopamine system through the ventral tegmental area. Brain Res 653: 278-284.

Di Chiara G (2000). Role of dopamine in the behavioural actions of nicotine related to addiction. Eur J Pharmacol 393: 295-314.

Donny EC, Caggiula AR, Knopf S, Brown C (1995). Nicotine selfadministration in rats. Psychopharmacology 122: 390-394.

Donny EC, Caggiula AR, Mielke MM, Jacobs KS, Rose C, Sved AF (1998). Acquisition of nicotine self-administration in rats: the effects of dose, feeding schedule, and drug contingency. Psychopharmacology 136: 83-90.

Donny EC, Caggiula AR, Mielke MM, Booth S, Gharib MA, Hoffman A et al (1999). Nicotine self-administration in rats on a progressive ratio schedule of reinforcement. Psychopharmacology 147: 135-142.

Donny EC, Chaudhri N, Caggiula AR, Evans-Martin FF, Booth S, Gharib MA et al (2003). Operant responding for a visual reinforcer in rats is enhanced by noncontingent nicotine: implications for nicotine self-administration and reinforcement. Psychopharmacology 169: 68-76.

Goldberg SR, Spealman RD, Goldberg DM (1981). Persistent behavior at high rates maintained by intravenous self-administration of nicotine. Science 214: 573-575.

Gonon FG (1988). Nonlinear relationship between impulse flow and dopamine released by rat midbrain dopaminergic neurons as studied by in vivo electrochemistry. Neuroscience 24: 19-28.

Harvey DM, Yasar S, Heishman SJ, Panlilio LV, Henningfield JE, Goldberg SR (2004). Nicotine serves as an effective reinforcer of intravenous drug-taking behavior in human cigarette smokers. Psychopharmacology 175: 134-142.

Jaffe JH (1990). Trivializing dependence. Br J Addict 85: 1425-1427.

Laviolette SR, van der Kooy D (2003). The motivational valence of nicotine in the rat ventral tegmental area is switched from rewarding to aversive following blockade of the alpha7-subunitcontaining nicotinic acetylcholine receptor. Psychopharmacology 166: 306-313.

Laviolette SR, van der Kooy D (2004). The neurobiology of nicotine addiction: bridging the gap from molecules to behaviour. Nat Rev Neurosci 5: 55-65.

Liu X, Caggiula AR, Palmatier MI, Donny EC, Chaudhri N, Sved AF (2005). Reinforcement Enhancing Effect of Nicotine Depends on the Reinforcement Valence of Non-drug Stimulus, Program No. 1027.14. Society for Neuroscience: Washington, DC.

Mansbach RS, Chambers LK, Rovetti CC (2000). Effects of the competitive nicotinic antagonist erysodine on behavior occasioned or maintained by nicotine: comparison with mecamylamine. Psychopharmacology 148: 234-242.

Mansvelder HD, Keath JR, McGehee DS (2002). Synaptic mechanisms underlie nicotine-induced excitability of brain reward areas. Neuron 33: 905-919.

Olausson P, Jentsch JD, Taylor JR (2003). Repeated nicotine exposure enhances reward-related learning in the rat. Neuropsychopharmacology 28: 1264-1271.

Olausson P, Jentsch JD, Taylor JR (2004). Repeated nicotine exposure enhances responding with conditioned reinforcement. Psychopharmacology 173: 98-104.

Palmatier MI, Caggiula AR, Liu X, Chaudhri N, Donny EC, Booth S et al (2006). Dissociating the primary reinforcing and reinforcement enhancing effects of nicotine in self-administration with concurrently available drug and environmental reinforcers. Psychopharmacology 184: 391-400.

Picciotto MR, Corrigall WA (2002). Neuronal systems underlying behaviors related to nicotine addiction: neural circuits and molecular genetics. J Neurosci 22: 3338-3341.

Risner ME, Goldberg SR (1983). A comparison of nicotine and cocaine self-administration in the dog: fixed-ratio and progressive-ratio schedules of intravenous drug infusion. J Pharmacol Exp Ther 224: 319-326. 
Rose JE (2006). Nicotine and nonnicotine factors in cigarette addiction. Psychopharmacology 184: 274-285.

Rose JE, Behm FM, Westman EC, Bates JE, Salley A (2003). Pharmacologic and sensorimotor components to satiation in cigarette smoking. Pharmacol Biochem Behav 76: 243-250.

Rose JE, Behm FM, Westman EC, Johnson M (2000). Dissociating nicotine and nonnicotine components of cigarette smoking. Pharmacol Biochem Behav 67: 71-81.

Rose JE, Levin ED (1991). Inter-relationships between conditioned and primary reinforcement in the maintenance of cigarette smoking. Br J Addict 86: 605-609.

Schilström B, Rawal N, Mameli-Engvall M, Nomikos GG, Svensson TH (2003). Dual effects of nicotine on dopamine neurons mediated by different nicotine receptor subtypes. Int $J$ Neuropsychopharm 6: 1-11.

Shoaib M, Schindler CW, Goldberg SR (1997). Nicotine selfadministration in rats: strain and nicotine pre-exposure effects on acquisition. Psychopharmacology 129: 35-43.

USDHHS (1988). Nicotine addiction: a report of the surgeon general. US Department of Health and Human Services, Office of the Assistant Secretary for Health, Office on Smoking and Health, Rockville, MD.

Wise RA (1987). Intravenous drug self-administration: a special case of positive reinforcement. In: Bozarth MA (ed). Methods of Assessing the Reinforcing Properties of Abused Drugs. SpringerVerlag: New York. pp 117-141. 